\title{
Practice-changing publications in functional urology: A case-based approach
}

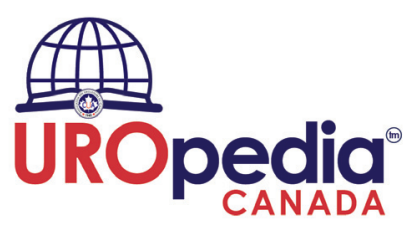

\author{
Laura N. Nguyen, $M D^{\prime}$; Ashley Cox, $M D^{2}$ \\ 'Division of Urology, McMaster University, Hamilton, ON, Canada; ${ }^{2}$ Department of Urology, Dalhousie University, Halifax, NS, Canada
}

Cite as: Nguyen L, Cox A. Practice-changing publications in functional urology: A case-based approach. Can Urol Assoc J 2021;15(7):E330-4. http://dx.doi.org/10.5489/cuaj.7468

The CUA has introduced a monthly webinar series called Practice-Changing Publications (PCP series), which features Canadian experts discussing recent data that have impacted their field. The CUAJ is proud to present summaries of these talks and the important research discussed in this series.

If you missed any of the webinars, you can access them at https://www.cua.org/UROpedia and earn all-important CPD Section 3 credits!

\section{Introduction}

The urological literature grows immensely by the day. Identifying practice-changing papers poses further difficulties. The purpose of this paper is to provide a common, case-based approach to applying the findings of some key papers in functional urology over the last 24 months. The cases described encompass: an elderly female with overactive bladder (OAB), a male patient with storage symptoms, a female patient with ulcerative interstitial cystitis, and a post-menopausal woman with recurrent urinary tract infections (UTI).

\section{Case 1}

A 76-year-old female presents with symptoms of urinary frequency, urgency, and urgency urinary incontinence (UUI). She voids 15 times per day and three times at night. She wears three pads per day for her UUI and denies stress urinary incontinence. She denies hematuria or pain and develops 1-2 UTIs per year. She has been prescribed an anticholinergic medication and she is concerned because she has read that it causes dementia.

\section{Anticholinergics and risk of dementia}

In 2020, Dmochowski et al published a systematic review and meta-analysis assessing the risk of dementia after $\geq 3$ months of anticholinergic medication use. ${ }^{1}$ English-language studies published before August 2019 were identified. Studies were included if they met the following criteria: 1) examined the impact of anticholinergic drug use for $\geq 3$ months on dementia or cognitive function in adult patients; 2) were a randomized control trial, case-control study, or cohort study; 3 ) contained an adequate description of the methods used; and 4) were a primary publication.

A total of 1990 articles were screened and 21 of these were included for the systematic review. Six studies met the criteria for being included in the meta-analysis for the outcome of dementia. The data from these studies represented 645865 patients across five countries. Five of the six studies reported an association between anticholinergic medications and incident dementia. All six of these studies were observational trials, with three being nested case-control studies. Followup duration ranged from 5.7-7.3 years in the cohort studies.

The meta-analysis found a rate ratio for incident dementia of 1.46 (95\% confidence interval [Cl] $1.17-1.81$; 95\% prediction interval [PI] 0.70-3.04). That is, the rate of dementia was $46 \%$ greater in patients exposed to anticholinergics compared to in patients not exposed to anticholinergics. The strength of the association increased with increased exposure to anticholinergics and was found when assessing studies looking at bladder medications specifically. This study suggested the risk should be considered in patients above and below the age of 65 and applied to both male and female patients.

The limitations of this study include the degree of heterogeneity between studies. Studies included in the meta-analysis were observational, with no randomized controlled trials. A time-to-dementia outcome was not assessed as part of the meta-analysis and, unfortunately, absolute rates of dementia were not provided to qualify the $46 \%$ relative increase in incident dementia cases.

This paper is significant, as it addresses an issue commonly brought forward by patients and summarizes a large body of literature that assesses the association between anticholinergic agents and risk of dementia. 
Case cont'd: Following a discussion with the patient on anticholinergic use and risk of dementia, you offer your patient a trial of mirabegron. She wants to know if it is safe for someone her age to take this class of medication.

\section{Mirabegron use in the elderly}

The PILLAR trial was recently published in European Urology. In this phase 4, double-blind, randomized, placebo-controlled trial, the efficacy, safety, and tolerability of mirabegron was assessed in patients aged 65 and over with urgency urinary incontinence. ${ }^{2}$ Patients were randomized to mirabegron $25 \mathrm{mg}(\mathrm{n}=445)$ daily vs. placebo $(\mathrm{n}=443)$ for 12 weeks. After four or eight weeks, the dose could be increased to $50 \mathrm{mg}$ daily. Patients were stratified by age $<75$. The co-primary endpoints were: 1 ) change from baseline to end of treatment (EOT) in mean number of voids/24 hours; and 2) incontinence episodes/24 hours.

A statistically significant improvement was found for mirabegron vs. placebo for both co-primary endpoints. Mirabegron resulted in a decrease in mean number of voids per 24 hours $(-0.7[0.2] ; 95 \% \mathrm{Cl}-1.0,-0.3)$ and a decrease in number of urgency incontinence episodes per 24 hours $(-0.6[0.1] ; 95 \% \mathrm{Cl}-0.8,-0.3)$. A larger proportion of patients in the mirabegron group achieved a $50 \%$ decrease in the number of incontinence episodes per 24 hours, $(72 \%$ vs.60\%, odds ratio $[\mathrm{OR}] 1.78,95 \% \mathrm{Cl} 1.32,2.38)$. However, for patients over the age of 75 , the improvement in incontinence episodes was diminished.

In terms of safety, the most common treatment-emergent adverse events (TEAEs) in the mirabegron group were UTIs, headaches, and diarrhea. TEAEs were reported in $47 \%$ of the mirabegron group and $39 \%$ of the placebo group. Cardiac disorders were reported in $2 \%$ of patients in the mirabegron group and $1.1 \%$ in the placebo group. Overall, mirabegron was concluded to be effective and safe in patients over 65 years of age.

Case cont'd: As the patient is about to leave your office, she also wants to know whether or not mirabegron is the only medicine in this class of bladder medications.

\section{Vibegron for the treatment of $O A B$}

Vibegron (Gemtesa ${ }^{\circledR}$ ) is a novel, highly selective, $B_{3}$-agonist. It has a long half-life (25-38 hours) and does not inhibit CYP2D6, limiting its drug-drug interactions. Phase 2 and 3 studies have found vibegron to be safe and effective for the treatment of OAB. ${ }^{3,4}$ The EMPOWUR trial assessed the safety and efficacy of vibegron in patients with OAB. This was an international, phase 3, randomized, double-blind, placebo- and active-controlled study. ${ }^{5} \mathrm{~A}$ total of 1518 patients were randomized to vibegron $75 \mathrm{mg}$ daily vs. tolterodine
$4 \mathrm{mg}$ daily vs. placebo for 12 weeks. The primary endpoints assessed were change from baseline to week 12 for: 1 ) mean number of voids/24 hours; and 2) mean number of UUI episodes/24 hours. Statistical analysis was carried out to compare vibegron to placebo and tolterodine to placebo, but vibegron was not directly compared to tolterodine.

Vibegron resulted in a statistically significant decrease in the mean number of voids/24 hours compared to placebo (least square [LS] mean difference of $-0.5,95 \% \mathrm{Cl}-0.9$, $-0.2, \mathrm{p}<0.001)$, whereas tolterodine did not when compared to placebo (LS mean difference $-0.3,95 \% \mathrm{Cl}-0.6,0.1$, $\mathrm{p}=0.0988$ ). When compared to placebo, both vibegron and tolterodine were found to statistically significantly reduce UUI episodes in a 24-hour period (vibegron LS mean difference of $-0.6,95 \% \mathrm{Cl}-0.9,-0.3, \mathrm{p}<0.0001$; tolterodine LS mean difference of $-0.4,95 \% \mathrm{Cl}-0.7,-0.1, \mathrm{p}=0.0123)$.

Vibegron was well-tolerated, with $1.7 \%$ of patients stopping the medication due to adverse events. This was in comparison to $1.1 \%$ in the placebo group and $3.3 \%$ in the tolterodine group. Hypertension developed in $1.7 \%$ of the vibegron group, $1.7 \%$ of the placebo group, and $2.6 \%$ of the tolterodine group.

Vibegron was approved by the FDA for the treatment of $\mathrm{OAB}$ as of December 2020 but is not yet approved by Health Canada.

Case conclusion: You inform the patient that, yes, mirabegron is the only option for her in this medication class for now.

\section{Case 2}

A 67-year-old male presents with frequency every 1-2 hours, urgency, and UUI. He has bothersome nocturia and mild voiding symptoms, but he describes his flow as satisfactory since starting tamsulosin $0.4 \mathrm{mg}$ daily five years ago. Physical exam reveals a mildly enlarged, smooth, non-tender prostate. Urinalysis is normal. You consider adding a second medication to target his storage symptoms but have some hesitation due to concerns that this will aggravate voiding symptoms or impair emptying.

\section{Mirabegron for the treatment of men with $O A B$}

Two large, randomized, placebo-controlled trials were published in 2020 demonstrating both efficacy and safety of the addition of mirabegron to tamsulosin for management of storage symptoms in male patients. ${ }^{6-8}$ The MATCH study included Japanese and Korean men treated with tamsulosin for benign prostatic hyperplasia (BPH). ${ }^{6}$ All study participants received tamsulosin $0.2 \mathrm{mg}$ plus one placebo tablet once daily for a four-week, single-blind screening period, followed by either for tamsulosin $0.2 \mathrm{mg}$ plus one placebo tablet (in the control group) or tamsulosin $0.2 \mathrm{mg}$ plus mirabegron 
$50 \mathrm{mg}$ (in the treatment group) for 12 weeks. The primary study endpoint was change in mean number of daily micturitions from baseline as measured by a three-day voiding diary. Secondary endpoints included change from baseline in: mean volume voided (MVV), mean number of urgency episodes, mean number of urge incontinence episodes, mean nocturia episodes, and patient-reported questionnaire scores related to voiding and overall function. Patients were followed for any TEAEs. After screening, 565 patients were randomized. Patients had a mean age of approximately 65 years, and the majority (95\%) were Japanese. Mean prostate volume was $31 \mathrm{~mL}$, and most patients had been symptomatic over 12 months.

After 12 weeks, the treatment group had a decrease of 1.27 (95\% Cl -1.65 to -0.89$)$ micturitions per 24 hours compared to a decrease of $0.75(95 \% \mathrm{Cl}-1.13$ to -0.38$)$ in the control group, a statistically significant difference. Of the secondary voiding diary endpoints, only change in MVV reached statistical significance, although non-significant improvement was seen in all other parameters. All patient-reported questionnaire scores showed statistically significant differences favoring the treatment group. TEAEs were uncommon and similar between groups; only three patients in the treatment group had an increase in postvoid residual (compared to one in the control group) and no patients required catheterization. ${ }^{6}$

The PLUS study was a multicenter, randomized, doubleblind, placebo-controlled study carried out in North America and Europe. ${ }^{7,8}$ The study began with a four-week, open label, run-in period during which all patients received tamsulosin $0.4 \mathrm{mg}$ daily. This was followed by randomization to either tamsulosin $0.4 \mathrm{mg}$ plus mirabegron $25 \mathrm{mg}$ daily for four weeks, then tamsulosin $0.4 \mathrm{mg}$ plus mirabegron $50 \mathrm{mg}$ daily for eight weeks (in the treatment group), or tamsulosin $0.4 \mathrm{mg}$ plus placebo daily for 12 weeks (in the control group). Similar to the MATCH study, the primary endpoint was change in the mean number of micturitions per day and secondary endpoints also included other voiding diary parameters (MVV, urgency episodes, total urgency and frequency score [TUFS], incontinence episodes) and questionnaire scores. TEAEs were also tracked, with attention paid to cardiovascular side effects and changes in voiding (peak flow and postvoid residual). After screening, 715 patients were randomized and 676 patients were including in the final analysis. The average patient age was approximately 65 years, with just over half of patients older than 65 .

The PLUS study demonstrated a small but statistically significant difference in the mean number of micturitions per day $(-2.00$ vs -1.62 , adjusted mean difference $-0.39,95 \%$ $\mathrm{Cl}-0.76,-0.02, \mathrm{p}=0.039)$. Statistically superior results were also seen in MVV, urgency episodes, and TUFS, along with non-significant trends towards improvement in incontinence episodes and International Prostate Symptom Score (IPSS). TEAEs were less common overall in the treatment group, with low and similar rates of serious TEAEs between groups. Cardiac TEAEs were uncommon, and hypertension occurred more frequently in the control group (11 patients vs. six patients). Urinary retention, as defined at the investigator's discretion with no specific need for catheterization, occurred in one patient in the control group and six in the treatment group, with only two patients in the treatment group requiring catheterization. ${ }^{7,8}$

The MATCH and PLUS trials showed very low risk of adverse events, and in particular very low rates of urinary retention or catheterization. While improvement in voiding parameters based on voiding diaries were small, change in MVV did reach statistical significance in both studies, and both trials reported significant change in subjective symptoms based on a variety of questionnaires.

Case conclusion: Based on the data from these trials, you confidently prescribe mirabegron $50 \mathrm{mg}$ for the case patient. Over the next few months, his storage symptoms improve significantly, he has no adverse events, and his postvoid residual remains low.

\section{Case 3}

A 70-year-old female presents with severe bladder pain, frequency, and nocturia. She voids 20 times per day and four times at night. She denies incontinence, gross hematuria, or infections. Her bladder capacity is 100 cc. Her last three urine cultures have been negative. Her last urologist diagnosed her with interstitial cystitis (IC)/bladder pain syndrome (BPS) and she wants to know if she has ulcers in her bladder.

\section{IC/BPS with Hunner lesions}

Recently, Lai et al sought to determine if there are any differences between patients with IC/BPS with and without Hunner lesions (ulcers, HL) based on their demographics, clinical presentation, comorbidities, urinary marker profiles, and treatment responses. ${ }^{9}$ In a systematic review and meta-analysis, 237 articles were identified, of which 59 were included in the systematic review and 23 in the meta-analysis.

The authors found that compared to IC/BPS patients without $\mathrm{HL}$, patients with $\mathrm{HL}$ were significantly older (mean difference [MD] 6.7 years, 95\% Cl 2.0-11.3, $\mathrm{p}=0.005)$, voided more frequently (MD 3.2 per day, $95 \% \mathrm{Cl} 1.1-5.4$, $\mathrm{p}=003$ ), had worse nocturia (MD 1.0 per night, $95 \% \mathrm{Cl}$ 0.1-2.0, $p=0.034)$, had higher Interstitial Cystitis Symptom Index scores (MD 2.2, 95\% Cl 1.4-3.0, $\mathrm{p}<0.001$ ), and lower cystometric capacity on urodynamics (MD -113 cc, 95\% $\mathrm{Cl}-164$ to $-61, \mathrm{p}<0.001)$. There was no difference in pain scores, symptom duration, or sex between patients with $\mathrm{HL}$ and without HL. Patients with HL were found to have higher 
levels of pro-inflammatory markers in their urine, including cytokines/chemokines and luminal nitric oxide. In terms of treatment, comparative studies were identified that showed a better response to oral cyclosporin $\mathrm{A}$ in those with HL vs. those without HL.

It was concluded that there are significant differences in these two populations, reinforcing the theory that patients with and without HL do represent two distinct clinical phenotypes. Despite these findings, patients still require a cystoscopy to definitively diagnose HL.

Case cont'd: You suspect this patient has a high chance of having HLs (older age; severe frequency, nocturia, and decreased bladder capacity). As recommended by the Canadian Urological Association guideline, ${ }^{10}$ you perform a cystoscopy under local anesthetic, which reveals a reddened mucosal area with small vessels radiating toward a central scar with a fibrin deposit in the middle, consistent with a HL. ${ }^{9}$

The patient wants to know: how you are going to remove these lesions? Will they recur? And if so, when?

\section{Treatment of Hunner lesions}

A recent randomized controlled trial compared the efficacy between transurethral coagulation and transurethral resection of $\mathrm{HL}$ in IC/BPS patients. ${ }^{11}$ They sought to evaluate whether resection would completely eliminate the HL. One hundred and twenty-six patients were randomized to transurethral resection (TUR, $n=64$ ) vs. transurethral cauterization (TUC, $\mathrm{n}=62$ ). This was a single-surgeon study. A resectoscope with bipolar loop or rollerball with continuous flow was used. Biopsies were taken at the time of TUC in all patients. Interestingly, two specimens revealed underlying malignancy. Patients were followed up at one, three, six, and 12 months postoperatively. Patients underwent cystoscopy at three and 12 months routinely and if they became symptomatic.

The primary outcome was recurrence-free time after treatment, where recurrence was defined as a return of symptoms in the presence of new HL on cystoscopy. There was no statistically significant difference in the recurrence-free time between the TUR and TUC groups (12.2 vs. 11.5 months, $\mathrm{p}=0.735$ ). Authors did not identify any risk factors for recurrence and there was no difference in the patient's voiding diaries or pain severity at any point in followup, although both groups improved from baseline. Importantly, there were more complications in the TUR group, with five $(7.9 \%)$ vs. two $(3.4 \%)$ bladder perforations in the TUR group vs. the TUC group.

Case conclusion: Based on this recent evidence, you explain to the patient that you would recommend TUC of the ulcers, which is safer than TUR, and will likely prevent the ulcers from returning for approximately 11-12 months.

\section{Case 4}

A 60-year-old, post-menopausal female presents with recurrent UTIs. Her symptoms are frequency, urgency, dysuria, and change in urine odor. She denies gross hematuria, fevers, or flank pain. She has had four symptomatic, culture-proven UTIs in the past six months. She is sexually active and describes vaginal dryness, pruritis, and dyspareunia. Her past medical history is notable for stage 1 estrogen receptor (ER)positive breast cancer with no lymph node involvement. She is on tamoxifen. You note vulvovaginal atrophy on pelvic exam. Her urinalysis is positive for white blood cells, leukocyte esterase, and red blood cells. A culture is sent.

You would like to start vaginal estrogen for UTI prevention and her genitourinary syndrome of menopause, but she is concerned about the effect of this on her ongoing breast cancer treatment.

Vaginal estrogen remains the mainstay of evidence-based UTI prevention in post-menopausal women. ${ }^{12,13}$ Despite evidence that systemic absorption is negligible with the use of topical vaginal estrogen for genitourinary syndrome of menopause (GSM), physicians continue to be hesitant to give vaginal estrogen to women, particularly those with history of previous or ongoing breast cancer. ${ }^{14}$

\section{Vaginal estrogen in patients with a history of breast cancer}

A recent review on the management of GSM in female cancer patients from the American Journal of Obstetrics and Gynecology 15 stratifies patients as "more desirable" or "less desirable" candidates for vaginal hormonal therapy. This is based on stage and grade of disease, hormone receptor status, current endocrine therapy, risk of recurrence, time since diagnosis, and effect on quality of life. The review also summarizes characteristics of options for local vaginal hormonal treatment and provides recommendations for conservative, non-hormonal treatment options for GSM. A large and comprehensive table also provides recommendations for local hormone therapy based on type of malignancy and patient/disease characteristics. From this table, for ER-positive breast cancer patients, local hormone therapy is an option if on tamoxifen, although caution is recommended for patients on aromatase inhibitors. For triple-negative breast cancer patients, local hormone therapy is also an option, though data is more limited. ${ }^{15}$

Case cont'd: Despite being ER-positive, this patient has lowstage disease with no lymph node involvement, is on tamoxifen, is very bothered by her symptoms of GSM/UTIs and, therefore, she stratifies as a "more desirable" candidate for local hormonal therapy. You counsel her appropriately and start her on vaginal estrogen with Vagifem 10 mcg to be inserted vaginally daily for three weeks, then twice weekly thereafter. 
You offer her empiric antibiotic treatment for her current UTI symptoms and grossly positive urine analysis, but she prefers to wait until the culture results are available. She is concerned about antibiotic side effects. A few days later, urine culture grows pan-sensitive E. coli.

\section{Antibiotic selection for uncomplicated UTls}

A systematic review and meta-analysis published in the Journal of Urology compared the efficacy and safety of fosfomycin to other antibiotics in women with acute, uncomplicated UTIs. ${ }^{16}$ A total of 539 articles were screened, of which 15 were included in the meta-analysis, with a total of 2295 patients. All trials compared a single dose of fosfomycin 3 $\mathrm{g}$ to another antibiotic (fluoroquinolones in five, trimethoprim or cotrimoxazole in three, nitrofurantoin in three, and beta-lactams in two). Fourteen studies were included in the microbiological eradication analysis, as they reported posttreatment cultures. Eleven studies were included for analysis of clinical cure, and eleven studies were included for analysis of safety outcomes. No significant difference was found in microbiological cure (OR 1.03, 95\% Cl 0.83-1.30, $\mathrm{p}=0.09$ ) or clinical cure in the meta-analysis (OR 1.16, 95\% $\mathrm{Cl} 0.91-1.49, \mathrm{p}=0.13)$. There was no difference in safety outcomes (OR 1.17, 95\% Cl 0.86-1.58, $\mathrm{p}=0.33$ ), although it was noted that most adverse effects reported for fosfomycin were "transient and short-lived." Single-dose fosfomycin was noted to have improved compliance and lower risk of antibiotic resistance, though this was not specifically reported in detail in this review. ${ }^{16}$

Case conclusion: Based on this study, you discuss treatment with fosfomycin. You treat her with one dose of fosfomycin $3 \mathrm{~g}$. Her symptoms resolve quickly and she has a long period following initiation of vaginal estrogen during which she is infection-free and symptom-free.

\section{Conclusions}

To summarize, we have presented recent literature in the field of functional urology to help with common clinical scenarios, including: anticholinergic medications and dementia risk, novel agents in $\mathrm{OAB}$ treatment, safety of mirabegron in the treatment of male lower urinary tract symptoms, ideal treatment of IC/BPS patients with $\mathrm{HL}$, and use of vaginal estrogen for the prevention of recurrent UTIs in women with a history of breast cancer. By relating these practice-altering papers to common clinical scenarios, their relevance and impact will hopefully be applied by many urologists in everyday practice.
Competing interests: Dr. Nguyen has been a speaker for Astellas and Pfizer. Dr. Cox has been an advisory board member for Astellas, Pfizer, and Teva; and has participated in a clinical trial supported by Aquinox.

\section{References}

1. Dmochowski RR, Thai S, Iglay K, et al. Increased risk of incident dementia following use of anticholinergic agents: A systematic literature review and meta-analysis. Neurourol Urodyn 2021;40:28-37. https://doi.org/10.1002/nau.24536

2. Wagg A, Staskin D, Engel E, et al. Efficacy, safety, and tolerability of mirabegron in patients aged $\geq 65$ yr with overactive bladder wet: A phase 4, double-blind, randomized, placebo-controlled study (PILLR). Eur Urol 2020;77:211-20. https://doi.org/10.1016/i.eururo.2019.10.002

3. Mitcheson HD, Samanta S, Muldowney K, et al. Vibegron (RVT-901/MK-4618/KRP-114V) administered once daily as monotherapy or concomitantly with tolterodine in patients with an overactive bladder: A multicenter, phase 2b, randomized, double-blind, controlled trial. Eur Urol 2019;75:274-82. h ttps://doi.org/10.1016/i.eururo.2018.10.006

4. Yoshida $M$, Takeda M, Gotoh $M$, et al. A novel potent and selective beta3-adrenoreceptor agonist, for the treatment of patients with overactive bladder: A randomized, double-blind, placebo-controlled phase 3 study. Eur Urol 2018;73:783-90. https://doi.org/10.1016/i.eururo.2017.12.022

5. Staskin D, Frankel J, Varano S, et al. International, phase 3, randomized, double-blind, placebo and active controlled study to evaluate the safety and efficacy of vibegron in patients with symptoms of overactive bladder: EMPOWUR. J Urol 2020;204:316-24. https://doi.org/10.1097/JU.0000000000000807

6. Kakizaki H, Lee KS, Yamamoto 0 , et al. Mirabegron add-on therapy to tamsulosin for the treatment of overactive bladder in men with lower urinary tract symptoms: A randomized, placebo-controlled study (MATCH). Eur Urol Focus 2020;6:729-37. https://doi.org/10.1016/i.euf.2019.10.019

7. Kaplan SA, Herschorn S, McVary KT, et al. Efficacy and safety of mirabegron vs. placebo add-on therapy in men with overactive bladder symptoms receiving tamsulosin for underlying benign prostatic hyperplasia: A randomized, phase 4 study (PLUS). J Urol 2020;203:1163-71. https://doi.org/10.1097/ JU.0000000000000738

8. Herschorn S, MCVary KT, Cambronero Santos J, et al. Mirabegron vs. placebo add-on therapy in men with overactive bladder symptoms receiving tamsulosin for underlying benign prostatic hyperplasia: A safety analysis from the randomized, phase 4 PLUS study. Urology 2021;147:235-42. https://doi.org/10.1016/i.urology.2020.09.040

9. Lai HH, Pickersgill NA, Vetter JM. Hunner lesion phenotype in interstitial cystitis/bladder pain syndrome: A systematic review and meta-analysis. J Urol 2020;204:518-23. https://doi.org/10.1097/ JU.0000000000001031

10. Cox A, Golda N, Nadeau G, et al. CUA guideline: Diagnosis and treatment of interstitial cystitis/bladder pain syndrome. Can Urol Assoc J 2016;10:E136-55. hitps://doi.org/10.5489/cuai.3786

11. Ko KJ, Cho WJ, Lee YS, et al. Comparison of the efficacy between transurethral coagulation and transurethral resection of Hunner lesion in interstitial cystitis/bladder pain syndrome patients: A prospective, randomized controlled trial. Eur Urol 2020;77:644-51. https://doi.org/10.1016/i.eururo.2020.01.002

12. Anger J, Lee $\mathrm{U}, \mathrm{Ackerman} \mathrm{AL}$, et al. Recurrent uncomplicated urinary tract infections in women: AUA/ CUA/SUFU guideline. J Urol 2019;202:282-9. https://doi.org/10.1097/JU.0000000000000296

13. Dason S, Dason JT, Kapoor A. Guidelines for the diagnosis and management of recurrent urinary tract infection in women. Can Urol Assoc J 2013;5:316-22. htrps://doi.org/10.5489/cuai.687

14. Krause M, Wheeler TL 2nd, Richter HE, et al. Systemic effects of vaginally administered estrogen therapy: A review. Female Pelvic Med Reconstr Surg 2010;16:188-95. https://doi.org/10.1097/ SPV.0b013e3181d7e86e

15. Crean-Tate KK, Faubion SS, Pederson HJ, et al. Management of genitourinary syndrome of menopause in female cancer patients: A focus on vaginal hormonal therapy. Am J Obstet Gynecol 2020;222:103-13. hitps://doi.org/10.1016/i.ajog.2019.08.043

16. Cai T, Tamanini I, Tascini C, et al. Fosfomycin trometamol vs. comparator antibiotics for the treatment of acute uncomplicated urinary tract infections in women: A systematic review and meta-analysis. J Urol 2020;203:570-8. https://doi.org/10.1097/JU.0000000000000620

Correspondence: Dr. Ashley Cox, Department of Urology, Dalhousie University, Halifax, NS, Canada; ashleycox@Dal.Ca 